\title{
Conhecimento das colaboradoras de uma instituição de sobre atendimento odontológico durante a gravidez
}

\author{
Knowledge of the employees of an institution about \\ dental care during pregnancy
}

\section{Wesley Lima Andrade', Ana Letícia de Lima Gonçalves², Éllen Maria Matos de Andrade², Daniela Nunes Nogueira ${ }^{4}$, Guereth Alexsanderson Oliveira Carvalho ${ }^{5}$, Neusa Barros Dantas Neta ${ }^{6}$}

\begin{abstract}
${ }^{1}$ Faculdade Integral Diferencial - Facid |Wyden. Teresina, Piauí, Brasil. ORCID: 0000-0002-8261-502X. wesleyfies10@hotmail.com ${ }^{2}$ Faculdade Integral Diferencial - Facid|Wyden. Teresina, Piauí, Brasil. ORCID: 0000-0002-7592-2056. aninhalg_@hotmail.com ${ }^{3}$ Instituto Superior de Educação de Caxias. Caxias, Maranhão, Brasil. ORCID: 0000-0001-7676-6956. ellenmaria.odonto@gmail.com ${ }^{4}$ Faculdade Integral Diferencial - Facid|Wyden. Teresina, Piauí, Brasil. ORCID: 0000-0003-0904-4694. daielanunes@hotmail.com 5Universidade Federal do Piauí. Teresina, Piauí, Brasil. ORCID: 0000-0003-3286-2943. guerethcarvalho@gmail.com ${ }^{6}$ Faculdade Integral Diferencial - Facid|Wyden. Teresina, Piauí, Brasil. ORCID: 0000-0001-7127-1463. nbdn2@msn.com
\end{abstract}

RESUMO | INTRODUÇÃO: Existem muitas crenças, tabus e mitos sobre o atendimento odontológico na gravidez. OBJETIVO: avaliar o conhecimento das colaboradoras sobre alterações bucais e necessidade de tratamento odontológico durante a gravidez. METODOLOGIA: Tratou-se de um estudo transversal. Foram incluídas na pesquisa mulheres colaboradoras ativas que trabalhavam na instituição de ensino em 2017. Foram excluídas da pesquisa colaboradoras que estivessem ausentes da faculdade na época. Os dados foram coletados por meio de entrevista através de questionário individual composto por questões objetivas que abordavam o conhecimento das colaboradoras, executada por um único avaliador, em seguida foi realizada a análise descritiva dos dados, apresentando-os com valores absolutos e porcentagens. RESULTADOS: Participaram da pesquisa 96 colaboradoras (taxa de resposta $88,9 \%$ ). Verificou-se que apenas $25 \%$ responderam corretamente todas as questões. $58,3 \%$ responderam que a gravidez causava problemas nos dentes. O problema mais citado foi sensibilidade dentária. Dentre as que responderam que poderia ser realizado algum procedimento, o procedimento mais relatado foi a limpeza dos dentes (91,7\%). CONCLUSÃO: É possível as grávidas se submeterem aos tratamentos odontológicos. Ainda são encontradas dificuldades para o atendimento odontológico da gestante.

PALAVRAS-CHAVE: Gestantes. Atendimento odontológico. Manifestações orais.
ABSTRACT | INTRODUCTION: There are many beliefs, taboos and myths about dental care in pregnancy. OBJECTIVE: to evaluate the collaborators' knowledge about oral changes and the need for dental treatment during pregnancy. METHODOLOGY: This was a cross-sectional study. Active female collaborators who worked at the educational institution in 2017 were included in the study. Collaborators who were absent from college at the time were excluded from the study. The data were collected through an interview through an individual questionnaire composed of objective questions that addressed the knowledge of female collaborators, performed by a single evaluator, then a descriptive analysis of the data was performed, presenting them with absolute values and percentages. RESULTS: Ninety-six female collaborators participated in the survey (response rate $88.9 \%$ ). It was found that only $25 \%$ correctly answered all the questions. $58.3 \%$ answered that pregnancy caused problems in the teeth. The most cited problem was dental sensitivity. Among those who answered that some procedure could be performed, the most reported procedure was tooth cleaning (91.7\%). CONCLUSION: It is possible for pregnant women to undergo dental treatments. Difficulties are still encountered in the dental care of pregnant women.

KEYWORDS: Pregnant women. Dental care. Oral manifestations. 


\section{Introdução}

Durante o curso de uma gravidez normal, várias lesões profundas e mudanças fisiológicas dinâmicas ocorrem tanto na mãe quanto no bebê em desenvolvimento. Neste período, diversas mudanças se manifestam, tanto hormonais como, cardíacas e respiratórias. Algumas das manifestações endócrinas e as alterações imunológicas induzidas pela gravidez aumentam a suscetibilidade da mãe a várias infecções, incluindo as da cavidade oral. Tais mudanças interferem no funcionamento do corpo e na personalidade, fazendo com que a mulher questione todo e qualquer procedimento, sendo muitas vezes influenciada também por crenças e mitos populares ${ }^{1}$.

Este questionamento ocorre principalmente relacionados aos procedimentos odontológicos. Várias organizações nacionais de saúde emitiram declarações nos últimos anos pedindo melhores cuidados de saúde bucal durante a gravidez. A literatura revela que o tratamento odontológico preventivo, diagnóstico e restaurador de rotina - incluindo terapia periodontal - durante a gravidez não aumenta os resultados adversos da gravidez ${ }^{2}$. Muitas gestantes ainda desconhecem as formas de prevenção e as possíveis alterações bucais que possam se manifestar durante 0 período gestacional e que podem prejudicar a saúde do futuro bebê ${ }^{3}$.

Pesquisas mostraram que mulheres grávidas são expostas a um maior risco de alterações gengivais. $O$ aumento da suscetibilidade a infecções na cavidade oral pode ocorrer devido à diminuição do pH e, consequentemente, a salivação capacidade tampão durante a gravidez, que, juntamente com a mudança de hábitos alimentares e de higiene bucal, contribui para crescimento bacteriano e aumenta o risco de cárie. Entre as alterações mais citadas na literatura são: granuloma piogênico, gengivite e periodontite ${ }^{4}$. Assim, o pré-natal odontológico nessa fase torna-se muito importante.

Uma das principais barreiras ainda encontradas para o atendimento de gestantes diz respeito à falta de motivação e muitas vezes o medo do profissional em atender pacientes grávidas. Em um estudo realizado no município de Fortaleza, 56,9\% $(n=70)$ dos entrevistados citaram a insegurança do profissional CD no manejo clínico. Além disso, existe a dificuldade de acesso aos serviços odontológicos públicos, tanto pela falta de vagas nas repartições públicas, quanto pela dificuldade de acesso ao atendimento privado ${ }^{5}$.

As mulheres ainda possuem um escasso conhecimento sobre as alterações bucais provocadas com a gravidez e sobre quais atendimentos podem receber nesse período. Dessa forma, o objetivo deste estudo foi avaliar o conhecimento das colaboradoras de uma instituição de ensino superior particular sobre alterações bucais e necessidade de tratamento odontológico durante a gravidez.

\section{Metodologia}

\section{Procedimentos éticos}

O estudo foi submetido ao Comitê de Ética em Pesquisa da Faculdade Integral Diferencial - FACID e aprovado sob número de protocolo CAAE: 57359316.0.0000.5211. As participantes assinaram o Termo de Consentimento Livre e Esclarecido (TCLE), que foi elaborado respeitando os critérios estabelecidos pela Resolução 466/12 do Conselho Nacional de Saúde (CNS), por se tratar de pesquisa envolvendo seres humanos.

O estudo apresentou risco de situações de constrangimento das participantes ao responder o questionário, porém, ele foi minimizado ao aplicá-los individualmente. Além disso, elas precisariam dispor de 12 minutos do seu tempo para responder o questionário. Todas as informações acerca desta pesquisa foram mantidas em sigilo, bem como o direito de retirar o consentimento a qualquer tempo e sem nenhum ônus.

\section{Tipo de pesquisa, cenário e participantes do estudo}

Tratou-se de um estudo transversal, realizado em uma Instituição de Ensino Superior Particular de Teresina-PI localizada na região leste da cidade. Ela possui 108 colaboradoras. A amostra do estudo foi do tipo censitária. 
Foram incluídas na pesquisa mulheres colaboradoras ativas que trabalhavam na instituição de ensino. Foram excluídas da pesquisa colaboradoras que estivessem ausentes da faculdade na época em que os questionários foram aplicados por motivos judiciais, problema de saúde e licença maternidade.

\section{Coleta dos dados}

Os dados foram coletados por meio de entrevista através de questionário indivudual elaborado para pesquisa. Ele foi composto por questões objetivas que abordavam o conhecimento das colaboradoras sobre: gravidez causar problemas nos dentes, possibilidade de realização de tratamentos dentários nas gestantes, o pré-natal odontológico, perda de dentes durante a gravidez, período mais adequado para o atendimento odontológico, parto prematuro devido aos problemas bucais e procedimentos odontológicos que podem ser realizados durante a gravidez. Os dados foram coletados em um tempo máximo de 12 minutos, por um único avaliador, no ano de 2017.

\section{Organização e análise dos dados}

Após a coleta de dados, as informações foram tabuladas e analisadas no programa SPSS para Windows versão 20.0. Realizou-se análise descritiva dos dados, apresentando-os com valores absolutos e porcentagens.

\section{Resultados}

Um total de 108 colaboradoras foram convidadas a participar e obteve-se 96 participações (taxa de resposta $=88,9 \%)$. As participantes tinham entre 18 e 58 anos de idade, com média de $34,7( \pm 10,5)$ anos. O conhecimento das gestantes sobre cuidados odontológicos na gravidez foi obtido através das 11 questões que estivessem marcadas corretamente segundo um gabarito elaborado pelo pesquisador. Ao final, verificou-se que apenas $25 \%$ das colaboradoras responderam corretamente todas as questões. $58,3 \%$ responderam que a gravidez causava problemas nos dentes (Tabela 1). O problema mais citado foi sensibilidade dentária (38,5\%).

Tabela 1. Frequência das respostas das colaboradoras sobre os problemas que gestação pode ocasionar na boca $(n=96)$

\begin{tabular}{lll}
\hline & $n$ & $\%$ \\
\hline Gravidez pode causar problema nos dentes & 56 & 58,3 \\
Sim & 14 & 14,6 \\
Não & 26 & 27,1 \\
Não sei & & 30,2 \\
\hline Problemas que pode causar & 29 & 19,8 \\
Cárie (sim) & 19 & 38,5 \\
Sangramento gengival (sim) & 37 & 10,4 \\
Sensibilidade dentária (sim) & 10 & 25,0 \\
Tártaro (sim) & 24 & 24 \\
Perda de dente (sim) & 2 & 2 \\
\hline
\end{tabular}


Dentre as colaboradoras, $82,3 \%(n=79)$, responderam que gestantes podem realizar tratamento odontológico e 9,4\% (n=9). Destes, 6,1\% responderam que o tratamento odontológico pode causar doenças no feto e 3,3\% que o tratamento pode induzir o parto prematuro (Tabela 2).

Tabela 2. Frequência das respostas das colaboradoras sobre realização de tratamento odontológico na gravidez $(n=96)$

\begin{tabular}{lll}
\hline & $\mathrm{n}$ & $\%$ \\
\hline Gestantes podem fazer tratamento odontológico & & \\
Sim & 79 & 82,3 \\
Não & 9 & 9,4 \\
Não sei & 8 & 8,3 \\
\hline Porque não pode fazer o tratamento o odontológico* & 0,0 \\
Pode levar ao parto prematuro & 0 & 6,1 \\
Pode causar doenças no feto & 6 & 3,3 \\
Pode induzir o parto no momento do atendimento & 3 & \\
odontológico & & 0,0 \\
Bebê pode nascer com baixo peso & 0 &
\end{tabular}

A tabela 3 evidencia o conhecimento das participantes sobre pré-natal odontológico. Apenas $25,0 \%$ ouviram falar sobre ele e $43,80 \%$ responderam que o atendimento odontológico poderia ser realizado durante todo período gestacional.

Tabela 3. Frequência das respostas das colaboradoras sobre os problemas que gestação pode ocasionar na boca $(n=96)$

\begin{tabular}{lcc}
\hline & $\mathrm{n}$ & $\%$ \\
\hline Você já ouviu falar sobre pré natal odontológico & 24 & 25,0 \\
Sim & 72 & 75,0 \\
Não & 27 & 28,1 \\
Qual o período mais adequado para procurar atendimento odontológico & 13.3 \\
Primeiro Trimestre & 13 & 8,3 \\
Segundo Trimestre & 8 & 43,8 \\
Terceiro Trimestre & 42 & 5,2 \\
Todo Período Gestacional & 5 & 1,0 \\
Nenhum & 1 & \\
Segundo e Terceiro & & \\
\hline
\end{tabular}


A tabela 4 mostra o conhecimento sobre mitos durante a gravidez. 18,8\% das colaboradoras disseram que é normal perder dentes durante a gravidez e destas $14,6 \%$ informaram que o motivo é que a gravidez deixa os dentes fracos. Com relação à possibilidade de os problemas bucais desencadearem um parto prematuro, apenas $11,5 \%$ responderam corretamente que pode. Quando questionadas sobre a possibilidade de problemas bucais da mãe afetarem a saúde do bebê, apenas $16,7 \%$ responderam que não e $28,1 \%$ não sabiam responder.

Tabela 4. Frequência das respostas das colaboradoras sobre mitos durante a gravidez $(n=96)$

\begin{tabular}{lll}
\hline & $\mathrm{n}$ & $\%$ \\
\hline É normal perder dentes durante a gravidez & 18 & 18,8 \\
Sim & 40 & 41,7 \\
Não & 38 & 39,6 \\
Não Sei & 3,1 \\
\hline Por que podem perder os dentes* & 0,0 \\
A gravidez deixa os dentes moles & 3 & 14,6 \\
A gravidez deixa os dentes cariados, levando a perda dos mesmos & 0 & 1,0 \\
A gravidez deixa os dentes fracos & 14 & 11,5 \\
A gravidez dificulta a higienização dos dentes & 1 & 38,5 \\
\hline Os problemas bucais podem levar ao parto prematuro & 11 & 50,0 \\
Sim & 37 & 55,2 \\
Não & 48 & 16,7 \\
Não Sei & 53,1 \\
\hline A falta de cuidado com a saúde bucal durante a gestação pode afetar a saúde do bebê & 16 \\
Sim & 53 & 27 \\
Não & & \\
Não sei & & \\
\hline *n 18 & & \\
\end{tabular}

Quanto aos procedimentos que podem ser realizados durante a gravidez, 96,9\% responderam que nenhum procedimento poderia ser realizado e apenas 3,1\% responderam que sim. Dentre as que responderam positivamente, o procedimento mais relado foi a limpeza dos dentes (91,7\%) (Gráfico 1). Apenas 10,4\% das mulheres afirmaram que é possível exame radiográfico durante a gravidez, enquanto $21,9 \%$ delas achavam possível ser realizado procedimentos anestésicos durante a gestação (Gráfico 1).

Gráfico 1. Procedimentos que podem ser realizados na gestação

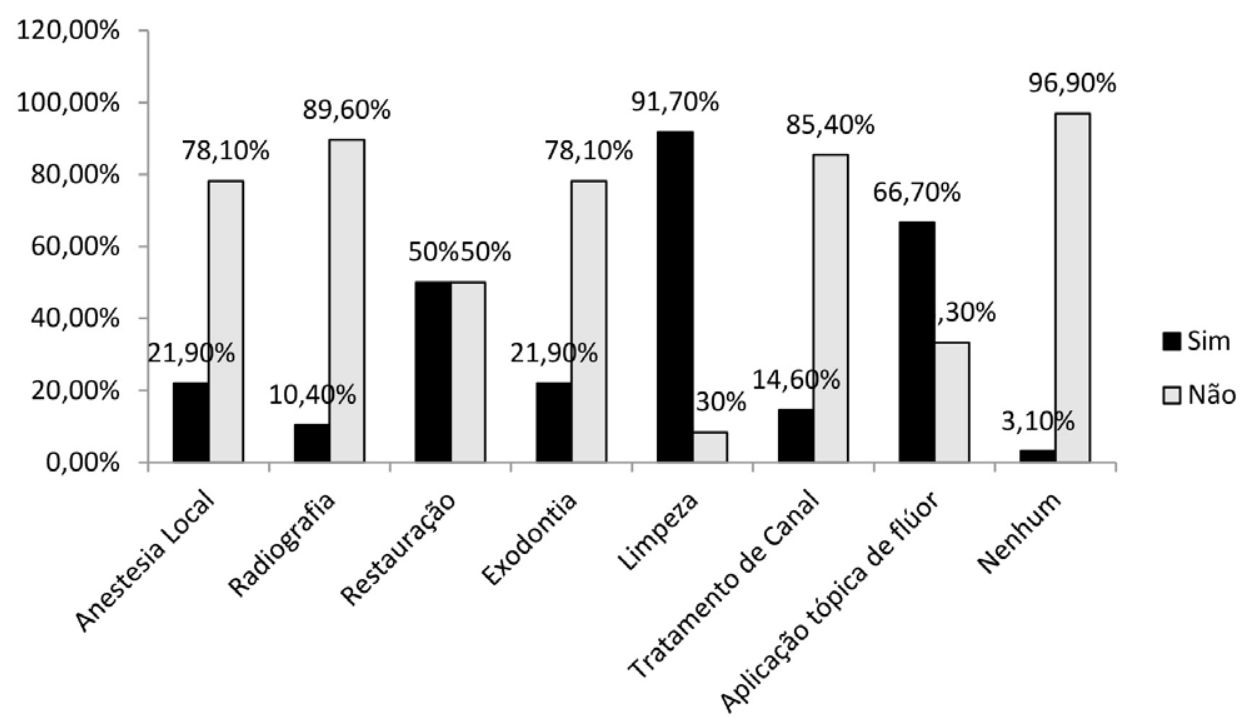




\section{Discussão}

Neste estudo, mais da metade das mulheres entrevistadas acreditavam que a gravidez pode acarretar problemas nos dentes, o que é errôneo. A gravidez, por exemplo, não causa gengivite. Acredita-se que as alterações hormonais é que influenciem a suscetibilidade à gengivite. Além disso, a negligência da mulher em relação à sua saúde bucal na gestação, é geralmente o principal fator responsável e podem acarretar patologias bucais, como a doença periodontal ${ }^{6,7}$. Gravidez não é a responsável pelo aparecimento de lesões cariosas e nem pela perda de minerais dos dentes da mãe ${ }^{7}$.

Os problemas relatados pelas entrevistadas como consequências da gestação foram, principalmente, sensibilidade dentinária e cárie dentária. Os resultados concordam com o realizado por Bastiani e seus colaboradores, que também observou que $48,75 \%$ das mulheres entrevistadas em seu estudo consideravam normal o aparecimento de cáries durante a gravidez, por acreditarem que o cálcio dos dentes seria transmitido ao feto. Tais dados demonstram, que mesmo nos dias atuais existem muitas crenças e mitos arraigados entre as mulheres, e as fazem acreditar que a gravidez pode acarretar problemas dentários como: cárie, sensibilidade, perda de dentes ou dentes mais frágeis ${ }^{3,8}$.

Quanto às possibilidades de tratamento odontológico em gestantes, a maioria acredita que é possível realizar procedimentos odontológicos no período gestacional, quando necessário. Tal achado confirma que, a maioria possuía conhecimento sobre esse aspecto. Corroborando com um estudo em que uma porcentagem igualmente alta, 83,8\%, de mulheres acreditam também que grávidas podem realizar tratamentos dentários durante a gravidez ${ }^{8}$. Gestantes podem realizar procedimentos odontológicos quando necessário, tendo-se em vista apenas a necessidade de alguns cuidados especiais no momento de atendê-las. Tais cuidados podem ser: sessões mais curtas de atendimento, minimizar dosagens de drogas anestésicas e utilizar agentes que sejam considerados os ideais para esse tipo especial de atendimento ${ }^{5,9}$.
Outro aspecto importante é a busca pelo dentista durante a gravidez. Cerca de $32,6 \%$ das mulheres não buscavam atendimento odontológico durante a gravidez por medo de acarretar problemas ao bebê. Em um estudo realizado em João Pessoa, 53,3\% das participantes grávidas relataram só procurar atendimento odontológico quando alguma coisa incomoda. Dentre os fatores relatados pelas mesmas estão medo, falta de tempo e descuido para não visitarem o cirurgião-dentista. Dentre as principais justificativas para o medo está o de haver danos à gestação e ao bebê. Estes dados corroboram com o presente estudo ${ }^{10}$. Tal crença deve ser desmitificada, através da educação sobre o tema, com realização de ações que possam elucidar o tema para as gestantes, para que, assim, as mulheres possam entender melhor a importância dos tratamentos odontológicos durante a gravide $z^{5}$.

Sobre pré-natal odontológico, os achados deste estudo encontraram uma taxa alta $(86,6 \%)$ de mulheres que desconheciam sobre o tema. Elas creem, inclusive, que os cuidados em saúde bucal no período gestacional são contraindicados. Isso pode ser justificado pelo fato de poucas gestantes procurarem os serviços odontológicos durante a época em que realizam o pré-natal, e apenas buscar atendimento em casos extremos de urgência, infecção ou dores ${ }^{3,5}$.

Ainda há uma resistência e insegurança das grávidas quanto à realização de um acompanhamento odontológico ${ }^{11}$. É importante salientar que a mãe possui um relevante papel dentro do núcleo familiar, um ponto chave na prevenção de doenças quando absorve os conceitos de saúde e introduz bons hábitos na família. O pré-natal poderia se apresentar de forma mais completa se as políticas de educação incluíssem a odontologia, encaminhando as gestantes para os consultórios odontológicos ${ }^{10}$. Portanto, 0 trabalho educativo com gestantes é a base da promoção da saúde para as gerações que estão por vir ${ }^{5}$. 
Dados apontam que $72,5 \%$ entrevistadas que também acreditavam ser possível manter a saúde bucal durante a gestação sem perder nenhum dente. Em contrapartida, muitas mulheres ainda consideram a perda de dentes durante a gestação um acontecimento comum, pois acreditam que o cálcio presente nos dentes da mãe é transferido todo para o bebê deixando os dentes mais fracos e frágeis. Além disso, elas acham que a gravidez pode levar ao aparecimento de cárie e assim, levar à necessidade de exodontias ${ }^{3}$.

O que se sabe é que a gravidez por si só não causa esses problemas, mas sim os hábitos adquiridos pelas gestantes, como o consumo excessivo de alimentos açucarados e diminuição no número de escovações devido aos quadros de enjoos matinais. Problemas como enjoos e sensibilidade gengival que ocorrem na gravidez devido as mudanças hormonais são fatores que ocasionam maior dificuldade para a gestante em realizar a correta higienização da boca, podendo ser um fator potencializador de problemas bucais ${ }^{12}$.

Quanto ao trimestre gestacional adequado para se submeter aos tratamentos odontológicos, uma pequena parcela afirmou corretamente que o período ideal é o segundo trimestre. Neste semestre, podem ser feitos procedimentos básicos e evitando-se procedimentos invasivos e demorados quando possível, sendo estes postergados para o pós-parto ${ }^{13}$.

O primeiro trimestre se apresenta como um momento muito delicado para que a mulher se submeta a procedimentos. Já o terceiro trimestre, é um período delicado e deve-se ter atenção redobrada, pois muitos fatores podem induzir ao parto. Assim, o segundo trimestre é período considerado ideal para que as grávidas procurem tratamento odontológico e possam ser submetidas a qualquer tipo de intervenção, que se caracteriza do quarto ao sexto mês de gestação ${ }^{14}$.

Embora o período ideal seja o segundo trimestre, quando se encontrar em uma situação de urgência, com dor ou infecção, o atendimento odontológico pode ser realizado a qualquer momento ${ }^{8}$. No primeiro trimestre deve-se ter cautela devido às transformações embriológicas do feto e o terceiro trimestre pelo fato de muitas mulheres apresentarem quadro de hipertensão, devendo-se assim evitar posições inclinadas pois podem levar a compressão da veia cava inferior podendo acarretar hipotensão e síncope ${ }^{15}$.
A doença periodontal pode ser classificada como um fator de risco para a indução de partos prematuros ou recém-nascidos que apresentem um baixo peso ${ }^{16}$. Com isso, é importante as gestantes conhecerem esses riscos para que elas possam fazer um acompanhamento buscando a prevenção contra a doença periodontal ${ }^{16}$. Doença periodontal pode ter influência no parto prematuro por funcionar como um reservatório para bactérias, que poderiam através do sangue atingir a cavidade uterina, bem como as bolsas periodontais aumentarem a produção de mediadores inflamatórios que seriam fontes de citocinas fetotóxicas, fatores estes que podem contribuir para o parto prematuro e baixo peso no recém-nascido ${ }^{17}$.

Na literatura há opiniões divergentes sobre problemas bucais que afetam a saúde do bebê. Não há ligação direta entre a saúde bucal da mãe e a do bebê, mas destacam que mesmo não se tendo a confirmação dessa informação deve-se prevenir possíveis problemas bucais ${ }^{10}$. Pode-se considerar que exista uma influência da saúde bucal da mãe na vida do bebê, pois existe uma possível relação entre a doença periodontal com o nascimento de bebês prematuros e com peso inferior ao ideal, tendo observado em seu estudo um total de $23 \%$ de mulheres que relataram um histórico de parto prematuro ${ }^{8,16,17}$.

Quanto aos procedimentos passíveis de serem realizados durante a gravidez, as respostas demonstram uma dificuldade das gestantes em relação ao conhecimento da possibilidade de se realizar todos os tipos de tratamento odontológico durante a gestação, apenas aplicar certos cuidados na hora das consultas para deixar a grávida confortável e confiante diante do profissional ${ }^{18}$.

Um dos maiores medos das mulheres durante a gravidez é se submeter a exames radiográficos. Os dados encontrados no presente estudo apontam que $53 \%$ das mulheres acreditavam que radiografias odontológicas não deveriam ser realizadas na gravidez ${ }^{3}$. Esse medo deve-se às informações distorcidas a respeito das radiografias. Quando realizadas com as precauções adequadas, os riscos de malefícios ao feto são mínimos. Além disso, a dose de radiação usada em Odontologia é mínima ${ }^{19}$. 
A anestesia se caracteriza como um meio seguro, devendo-se ter cuidado apenas para se utilizar lidocaína associada a vasoconstrictor, e respeitar as dosagens corretas dos anestésicos. Devem-se evitar anestésicos do tipo éster por causa da sua potencialidade de causar alergias ${ }^{3}$. Drogas como bupivacaína e mepivacaína também poderiam ser indicadas por terem menor chance de passagem placentária, entretanto a bupivacaína deve ser evitada por apresentar um tempo ação longo ${ }^{15}$.

Já a mepivacaína deve ser evitada devido a imaturidade do sistema hepático do feto e a articaína excluída por poder causar meteglobinemia. Anestésicos tópicos, como a benzocaína, também devem ser evitados por diminuirem a circulação placentária. Assim, preconiza-se como anestésico mais seguro a lidocaína $2 \%$ a $3 \%$, associada com adrenalina, no máximo 2 tubetes, observando o refluxo na seringa para evitar injeções intravasculares ${ }^{15}$.

O cuidado em saúde da gestante e da puérpera deve ser proporcionado por uma equipe multiprofissional. Desta forma, o cirurgião dentista deve se articular nos serviços de assistência pré-natal, garantindo um atendimento integral, humanizado e de qualidade. Para isso, é imprescindível que eles conheçam o cenário do cuidado em rede para a assistência à saúde da gestante e da puérpera, a rede cegonha. Esta política insere o dentista como profissional necessário ao atendimento básico na gestação. Além disso, elenca atribuições desse profissional que envolvem ações educativa, preventiva e curativa ${ }^{20}$.

Diante dos dados encontrados neste trabalho, observou-se que é necessário ampliar as discussões em torno do pré-natal odontológico e da integração da equipe de saúde bucal aos demais profissionais responsáveis pelo acompanhamento pré-natal. Assim, a formação em Odontologia precisa subsidiar e estimular a realização dessa prática, na dimensão promotora de saúde, preventiva e assistencial.

Ainda são encontradas dificuldades para o atendimento odontológico na gestação, devido à forte presença de crenças e mitos que devem ser trabalhados com melhor planejamento de programa de promoção da saúde bucal. Desta forma, salienta-se a importância da educação permanente nos serviços de saúde, para subsidiar o processo de trabalho da equipe de saúde, e fomentar a integração da mesma, fortalecendo o cuidado pré-natal com base na integralidade.

As principais limitações ao desenvolvimento deste trabalho foram o uso de amostra de conveniência, restrita aos dados-resultados das entrevistas com as mulheres participantes, passível de viés de informação, pois dependia da informação prévia adquirida por elas. Não obstante essas limitações, permitiram que entender o conhecimento prévio das participantes para o planejamento de ações e estratégias em saúde bucal, tanto para o setor público como para o setor privado, para desmistificar o assunto pré-natal odontológico. Com isso, sugere-se estudos voltados para aplicação de palestras sobre pré-natal odontológico e verificando o aprendizado das mesmas quanto as palestras.

\section{Considerações Finais}

As mulheres entrevistadas ainda estão desinformadas sobre as possíveis alterações bucais na gravidez, bem como, quanto aos procedimentos odontológicos que podem ser realizados e período ideal para o atendimento. Também não possuem conhecimento sobre o que é o pré-natal odontológico.

A maioria das mulheres não sabe que a doença periodontal pode levar ao parto prematuro e ao nascimento de bebês com baixo peso.

Medidas educativas devem ser sugeridas para maior conscientização das mulheres em período gestacional, para que elas procurem realizar o pré-natal odontológico, e assim, compreendam a importância do tratamento odontológico durante a gravidez para sua saúde e a do bebê. 


\section{Contribuições dos autores}

Andrade WL e Gonçalves ALL realizaram a revisão de literatura e análise de dados e escreveram o artigo. EMMA realizou a análise e discussão dos resultados. Nogueira DN criou o desenho do estudo, realizou a análise dos resultados e avaliação crítica. Dantas Neta NB criou o desenho do estudo e realizou a análise e discussão dos resultados, avaliação crítica e orientação para o trabalho. Carvalho GAO realizou a análise e discussão dos resultados.

\section{Conflitos de interesses}

Nenhum conflito financeiro, legal ou político envolvendo terceiros (governo, empresas e fundações privadas, etc.) foi declarado para nenhum aspecto do trabalho submetido (incluindo mas não limitando-se a subvenções e financiamentos, participação em conselho consultivo, desenho de estudo, preparação de manuscrito, análise estatística, etc.).

\section{Referências}

1. Figueiredo CSA, Rosalem CGC, Cantanhede CAL, Thomaz ÉBAF, Cruz MCFN. Systemic alterations and their oral manifestations in pregnant women. J Obstet Gynaecol Res. 2017 Jan;43(1):16-22. doi: 10.1111/jog.13150

2. Steinberg BJ, Hilton IV, Iida H, Samelson R. Oral health and dental care during pregnancy. Dent Clin North Am. 2013 Apr;57(2):195-210. doi: 10.1016/j.cden.2013.01.002

3. Bastiani C, Cota ALS, Provenzano MGA, Fracasso MLC, Honório HM, Rios D. Conhecimento das gestantes sobre alterações bucais e tratamento odontológico durante a gravidez. Odontol Clín-Cient 2010;9(2):155-60.

4. Jain K, Kaur H. Prevalence of oral lesions and measurement of salivary $\mathrm{pH}$ in the different trimesters of pregnancy. Singapore Med J 2015;56(1):53-57. doi: 10.11622/smedj.2015010

5. Mendes-Júnior FIR, Bandeira MAM, Tajra FS. Perception by professionals on the relevance of the oral health indicators in a metropolis of the Brazilian northeastern. Saúde debate 2015 JanMar;39(104):147-58. doi: 10.1590/0103-110420151040205

6. Aguiar TC, Valsecki Júnior A, Silva SRC, Rosell FL, Tagliaferro EPS. Evaluation of risk factors suffering dental caries in pregnants from Araraquara, Brazil. Rev Cubana Estomatol 2011;48(4):341-51.

7. Kashetty M, Kumbhar S, Patil S, Patil P. Oral hygiene status, gingival status, periodontal status, and treatment needs among pregnant and nonpregnant women: A comparative study. J Indian Soc Periodontol. 2018 Mar-Apr;22(2):164-70. doi: 10.4103/jisp. jisp_319_17

8. Costa DLA, Carlos MX, Mota OML, Pereira SLS. Conhecimento de gestantes sobre a relação entre doenças periodontais e gravidez. Braz J Periodontol 2015;25(04):7-13.
9. Poletto VC, Stona P, Weber JBB, Fritsher AMG. Atendimento odontológico em gestantes: uma revisão da literatura. Stomatos 2008;14(26):64-75.

10. Nóbrega MTC, Freire JCP, Dias-Ribeiro E, Ghersel H, Ghersel ELA. Evaluation of pregnant women perception about caries and periodontal diseases. Arch Health Invest 2016;5(5):247-250. doi: 10.21270/archi.v5i5.1698

11. Oliveira EC, Lopes JMO, Santos PCF, Magalhães SR. Atendimento odontológico a gestantes: a importância do conhecimento da saúde bucal. Revista de Iniciação Científica da Universidade Vale do Rio Verde, Três Corações 2014;4(1):11-23.

12.Cabral MCB, Santos TS, Moreira TP. Percepção das gestantes do Programa de Saúde da Família em relação à saúde bucal no município de Ribeirópolis, Sergipe, Brasil. Rev Port Saúde Pública 2013;31(2):160-7. doi: 10.1016/j.rpsp.2013.05.004

13. Vamos CA, Thompson EL, Avendano M, Daley EM, Quinonez $\mathrm{RB}$, Boggess $\mathrm{K}$. Oral health promotion interventions during pregnancy: a systematic review. Community Dent Oral Epidemiol. 2015 Oct;43(5):385-96. doi: 10.1111/cdoe.12167

14. Hartnett E, Haber J, Krainovich-Miller B, Bella A, Vasilyeva A, Lange Kessler J. Oral Health in Pregnancy. J Obstet Gynecol Neonatal Nurs. 2016 Jul-Aug;45(4):565-73. doi: 10.1016/j. jogn.2016.04.005

15. Rodrigues F, Mármora B, Carrion SJ. Anestesia local em gestantes na odontologia contemporânea. J Heal Npeps 2017; 2(1): 254-71.

16. Domingues JM, Oliveira LCBS, Alves J, Machado W. A doença periodontal como possível fator de risco colaborador, dentre os demais fatores de risco clássicos, para o parto prematuro e/ou baixo peso ao nascer - revisão de literatura. Periodontia 2010;20(02):33-8.

17. George A, Shamim S, Johnson M, Ajwani S, Bhole S, Blinkhorn A et al. Periodontal treatment during pregnancy and birth outcomes: a meta-analysis of randomised trials. Int J Evid Based Healthc. 2011 Jun;9(2):122-47. doi: 10.1111/j.17441609.2011.00210.x

18. Martins LO, Pinheiro RDPS, Arantes DC, Nascimento LS, Santos Júnior PB. Assistência odontológica à gestante: percepção do cirurgião-dentistaDental care for pregnant woman: dental surgeon's perceptions. Rev Pan-Amaz Saude 2013;4(4):11-8. doi: 10.5123/S2176-62232013000400002

19. Codato LAB, Nakama L, Melchior R. Percepções de gestantes sobre atenção odontológica durante a gravidez. Ciênc saúde coletiva 2008;13(3):1075-80. doi: 10.1590/S1413$\underline{81232008000300030}$

20. Ferreira SMSP, Pinheiro ES, Silva RV, Silva JF, Batista LD, Fernandes CG. Prenatal dental care: accessibility and shares offered by primary care in Vitória da Conquista-BA. Faculdade de Odontologia de Lins/Unimep 2016;26(2):3-16. 